\title{
A 60-year review on the changing epidemiology of measles in capital Beijing, China, 1951-2011
}

\author{
Juan Li, Li Lu, Xinghuo Pang, Meiping Sun, Rui Ma, Donglei Liu and Jiang Wu*
}

\begin{abstract}
Background: China pledged to join the global effort to eliminate measles by 2012. To improve measles control strategy, the epidemic trend and population immunity of measles were investigated in 1951-2011 in Beijing.

Methods: The changing trend of measles since 1951 was described based on measles surveillance data from Beijing Centre of Disease Control and Prevention (CDC). The measles vaccination coverage and antibody level were assessed by routinely reported measles vaccination data and twenty-one sero-epidemiological surveys.

Results: The incidence of measles has decreased significantly from 593.5/100,000 in 1951 (peaked at 2721.0/100,000 in 1955), to $0.5 / 100,000$ in 2011 due to increasing vaccination coverage of 95\%-99\%. Incidence rebounded from 6.6/ 100,000 to 24.5/100,000 since 2005 and decreased after measles vaccine (MV) supplementary immunization activities (SIAs) in 2010. Measles antibody positive rate was 85\%-95\% in most of years since 1981. High-risk districts were spotted in Chaoyang, Fengtai and Changping districts in recent 15 years. Age-specific incidence and proportion of measles varied over time. The most affected population were younger children of 1-4 years before 1978, older children of 5-14 years in 1978-1996, infant of $<1$ years and adults of $\geq 15$ years in period of aim to measles elimination.

Conclusion: Strategies at different stages had a prevailing effect on the epidemic dynamics of measles in recent 60 years in Beijing. It will be essential to validate reported vaccination coverage, improve vaccination coverage in adults and strengthen measles surveillance in the anticipated elimination campaign for measles.
\end{abstract}

Keywords: Measles, Epidemiology, Incidence, Vaccine, Coverage, Sero-epidemiology

\section{Background}

Measles is a severe respiratory infectious disease caused by measles virus [1]. Measles has been statutorily notifiable since the earlier National Notifiable Diseases Reporting System (NNDRS) was established in 1950. Hospitals reported cases by posting a card to the county Center for Disease Control (CDC). Every month county CDC aggregated data that were then submitted through prefecture and provincial CDC to reach the national level [2]. In 1987 NNDRS was further improved, which reported basic epidemiologic data including age, sex, date of disease onset and residence by electronic document each month [3]. Since 2004 after severe acute respiratory syndrome (SARS), NNDRS was upgraded to direct reporting through network to improve timeliness, completeness of case reporting [4].

\footnotetext{
* Correspondence: wj81732@yahoo.com.cn

Beijing Center for Disease Control and Prevention, 16 Hepingli Middle Road, Dongcheng District, Beijing 100013, China
}

For each suspected case, the local CDC is required to carry out an epidemiological investigation, including obtaining specimens for laboratory confirmation since 2000.

Liquid measles vaccine (MV) was introduced in China in 1965. The 1978 establishment of the national Expanded Program on Immunization (EPI) resulted in a standard routine immunization schedule including MV administered as 1 dose to infants aged 8 months. In 1986, a 2dose schedule using lyophilized MV at 8 months and 7 years of age was implemented [5]. The next step was a 1997 national plan of action for accelerated measles control that called for $90 \%$ MV coverage, measles surveillance and catch-up campaigns for provinces. In 2005, the World Health Organization's (WHO) Regional Committee for the Western Pacific Region set a target date of 2012 for measles elimination in the region including China [6,7]. Since 2005, the age of administration of the second dose was lowered from 7 years to 1.5 years. The 2006-2012
C Biomed Central

(c) 2013 Li et al.; licensee BioMed Central Ltd. This is an open access article distributed under the terms of the Creative Commons Attribution License (http://creativecommons.org/licenses/by/2.0), which permits unrestricted use, distribution, and reproduction in any medium, provided the original work is properly cited. 
national action plan to eliminate measles claimed comprehensive measures including 95\% MV coverage of 2 doses, measles surveillance, regional and national supplementary immunization activities (SIAs), vaccination certificate of children at admission to child care setting and school $[8,9]$. China is a developing country with vast territory of 9.6 million square kilometers and large population of 1.37 billion, which has 23 provinces, 4 municipalities, 5 autonomous regions and 2 special administrative regions with different levels of economic and social development, predicting different challenges in reaching measles elimination. Hence, strategies of prevention and control measles were slightly different among different provinces.

Beijing is capital of China with a total area of 16,808 square kilometers. According to the 2010 national census, there were 19.6 million populations, of which 7 million migrants seek work in Beijing out of their province of origin. Referring to the historical periods of control of measles in China, combined with different strategies in Beijing, the strategies evolved from 1951 to 2011 in five stages (Figure 1):

Stage 1: Period prior to vaccination (1951-1965): Incidence of measles was in a natural infection state.

Stage 2: Initial period of measles immunization (19661977): MV was administered as 1 dose to infants aged 6 months. The second and third doses were administered to children of 4 and 8 years old in 1972. The age of administration of the first dose was increased to 8 months in 1974. Vaccine cold chain was not established. No refrigerant equipment was available and vaccine could not be stored at proper temperatures. In order to ensure vaccine immunogenicity, children with a certain age were gathered to vaccination twice each year in winter and spring.
Stage 3: Period of planned immunization (1978-1996): The 1978 establishment of the Beijing EPI resulted in a standard routine immunization schedule as a 2dose schedule at 8 months and 1.5 years of age. Since 1982, Jing ${ }_{55}$ MV strains were replaced by $\mathrm{Hu}_{191}$ strains to improve vaccine immunogenicity. MV immunization population was expanded to students in Grade 1 and 7 in school, freshman in college. In 1987, the change of MV dosage form from liquid to lyophilized improved vaccine stability. With the gradually improvement of vaccine cold chain, children could be vaccinated in the whole year with the establishment of medical institutions designated inoculation. The subsequent expansion of cold chain infrastructure allowed routine immunization to cover the entire country.

Stage 4: Accelerated period of measles control (19972004): Since 2000, SIAs among migrant children under 7 years old was implemented every year. Measles vaccination dose was increased from $0.2 \mathrm{ml}$ to $0.5 \mathrm{ml}$ to enhance vaccine immunogenicity in 2004.

Stage 5: Period of aim to measles elimination (20052011): Since 2005, the vaccination schedule was recommended 3 -doses vaccine schedule at 8 months, 1.5 and 6 years of age with univalent MV in all districts. Measles, rubella and mumps combined attenuated live vaccine (MMR) and measles and rubella combined attenuated live vaccine (MR) were introduced into the immunization program in 2006 and 2009, respectively. Measles vaccination campaign targeted migrant workers aged $15-40$ years was implemented every year. The synchronized nationwide SIAs targeting children aged 8 months to 14 years was conducted in 2010 .

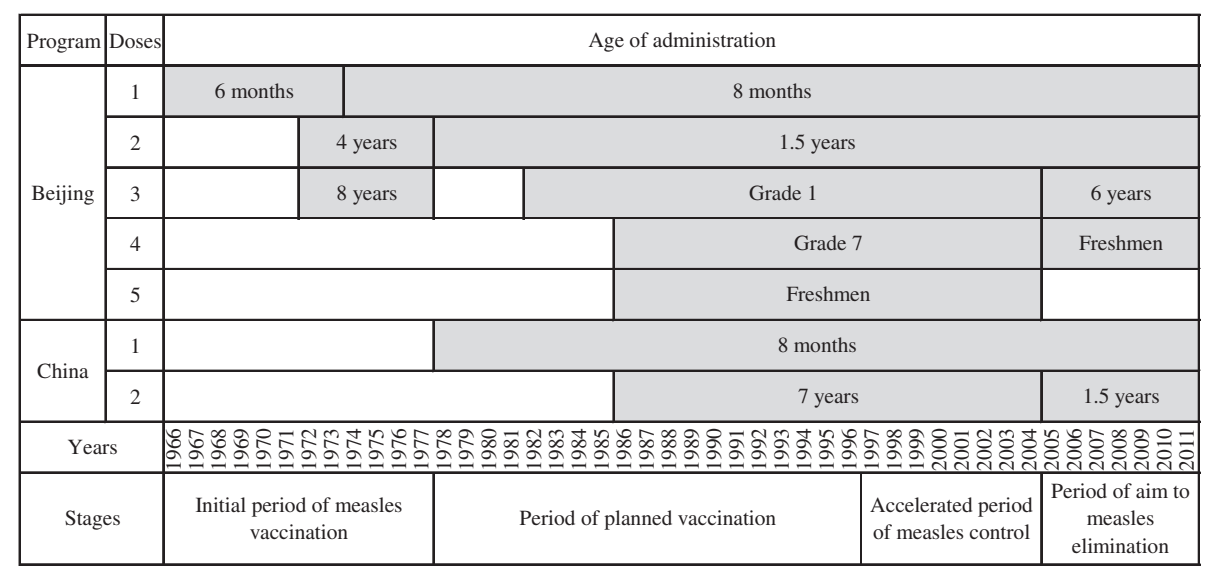

Figure 1 Measles vaccine immunization program of Beijing and China, 1966-2011. 
Global vaccination programs have led to a worldwide decrease in the occurrence of measles [10,11], with some areas documenting an interruption of transmission of the endemic virus $[12,13]$. This study aimed to review the changing epidemiology of measles, measles vaccination coverage in relationship to the trend of measles incidence, herd immunity of the population against measles through periodic sero-epidemiological surveys from 1951 to 2011 in Beijing.

\section{Methods}

\section{Case definition and source of data}

Data of measles cases and deaths in 1951-2011 were obtained from Beijing CDC. Suspected measles cases was diagnosed clinically as a person of any age with fever, rash and one of the following: cough, coryza or conjunctivitis, classified as either laboratory-confirmed, epidemiologically linked, clinically confirmed or discarded, by guidelines of World Health Organization [14,15]. With the improvement of surveillance and moving towards measles elimination, the demands of measles case classification have changed during different phases. Before period of planned vaccination in 1978, almost all measles were clinical diagnosed case due to lack of routine and convenient laboratory test method. Since 1980, laboratory test method of measles antibody was used in case diagnosis. The national serological surveillance laboratory network was established in 2001 to provide serological confirmation of measles infection and to support measles surveillance. All cases included in this analysis were confirmed cases including laboratory or clinical confirmed and epidemiological related cases. Annual incidence and morbidity (per 100,000 populations) were calculated based on Beijing census data reported by the statistical yearbook of Beijing.

\section{Vaccination coverage}

Administrative data based on report from health centre staff were the main sources of immunization coverage. The doses of vaccines administered in a given period (usually 1 month) were reported to prefecture CDC, in which data were collected and reported to Beijing CDC. In Beijing CDC, data were aggregated to estimate the average coverage rate using the numerator (the total number of actually immunized children) divided by the denominator (the total number of children who should be immunized by immunization schedule). For example, the 1978 establishment of the Beijing Expanded Program Immunization (EPI) recommended a standard routine immunization schedule as a 2-dose MV at age of 8 months and 1.5 years. The target population of coverage rate of first and second doses was children aged more than 9 and 19 months old, respectively, who lived in Beijing for more than 2 months. Vaccine immunization data from 1971 to 2011 were obtained from Beijing CDC.

\section{Sero-epidemiological surveys}

In order to assess the level of immunity of the population against measles, sero-epidemiological surveys were conducted in 1973-1977, 1979-1982, 1986, 1989, 1990, 1995-2000, 2002, 2003, and 2007 in selected districts of Beijing. The sampling procedure was based on a threestage protocol. Firstly, at least 3 prefectures were selected as random or specific survey sites from downtown, suburban and rural areas. Secondly, the villages were sampled by probability-proportional-to-size sampling (PPS). Thirdly, a number of objects per birth cohort were selected by convenience sampling method in the local population. Blood samples were collected from healthy persons with consent. Information integrated by the time of blood collection including basic demographic data, history of previous measles infection and MV vaccination. The blood samples were analyzed for measles IgG antibodies using measles enzyme immunoassay. Measles sero-epidemiology data from 1973 to 2007 were obtained from Beijing CDC. Seroepidemiology surveys were performed with the approval of Beijing CDC ethics committee.

\section{Statistical analyses}

We analyzed the data of confirmed measles cases reported in Beijing in 1951-2011 to identify epidemiological changing features, spatial geographic and age distribution, vaccination coverage and measles antibody positive rate. We used Microsoft Excel 2000, SPSS 17.0 and Mapinfo 8.5 for data analysis.

\section{Results}

\section{Incidence and mortality of measles in Beijing}

Overall, 1,507,956 reported cases and 11,130 deaths of measles were recorded in Beijing over the 60-year period. Figure 2 showed the temporal pattern of the incidence and mortality of measles in Beijing in 19512011. The overall declining trend was clear, with the annual average incidence decreasing from 593.5/100,000 in 1951 to $0.5 / 100,000$ in 2011 and mortality declining from $48.4 / 100,000$ in 1951 to 0 in 2011. From 1951 to 1965, measles incidence fluctuation ranged from 390.7/ 100,000 to $2721.0 / 100,000$. A frequent peak of incidence was observed every 2-3 years. Measles mortality was high, ranging from $2.5 / 100,000$ to $48.4 / 100,000$. In 1966-1977, incidence ranged from $32.2 / 100,000$ to 397.1/100.000, which declined $92.7 \%$ compared with incidence before vaccination. There was a 3-4 year periodic recurrent pattern. Mortality declined significantly to $0.1-1.5 / 100,000$. In 1978-1996, there were two phases in variation of incidence. Before 1985, average incidence was $78.7 / 100,000$. Since 1986, the incidence of measles has remained at a relatively low level, less than 2.0/ 100,000 in most of years. No measles deaths were reported from 1986 to 1996. In 1997-2004, incidence 


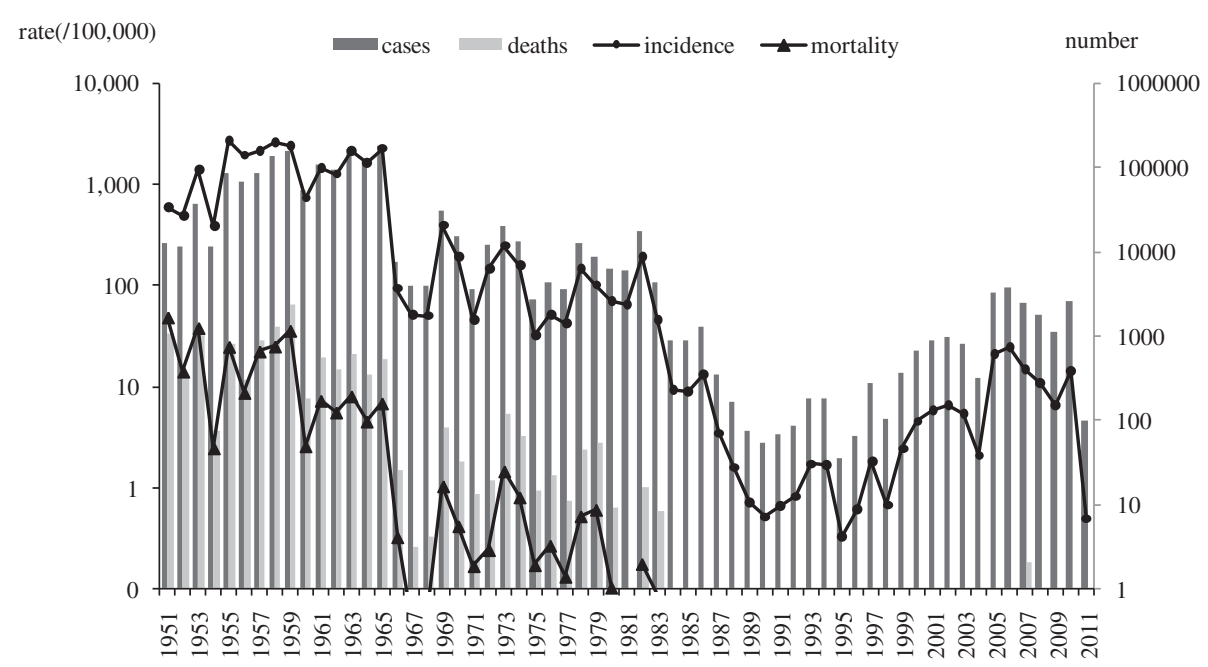

year

Figure 2 Measles incidence and morbidity in Beijing, 1951-2011.

was between $0.7 / 100,000$ and $6.6 / 100,000$. In 20052011, incidence rebounded from $6.6 / 100,000$ to $24.5 /$ 100,000 . After MV SIAs in 2010, incidence decreased to $0.5 / 100,000$ in 2011. 4 deaths were reported in 19972011.

\section{Age distribution of measles}

Age-specific incidence of measles varied over time. In 1957-1962 prior to vaccination, high measles incidence, $685.5 / 100,000$ to $14,743.2 / 100,000$, was found in children aged $\leq 14$ years, especially among children aged $1-2$

incidence $(/ 100,000)$

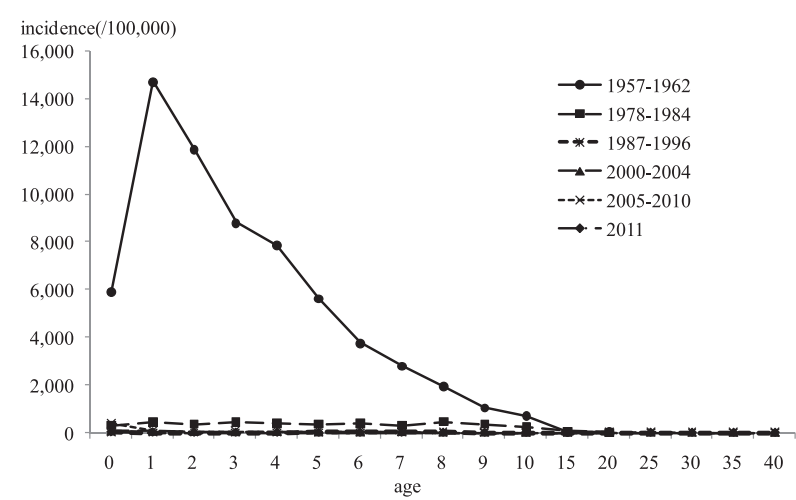

incidence $(/ 100,000)$

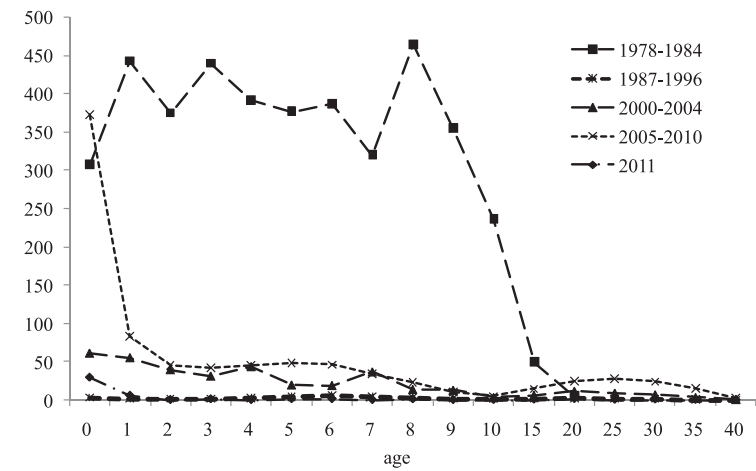

Figure 3 Age-specific incidence and proportion of measles in Beijing, 1957-2011. The measles cases by age groups in 1963-1972, 1985-1986 and 1997-1999 and the age-specific incidence in 1963-1977, 1985-1986 and 1997-1999 were not recorded in detail.
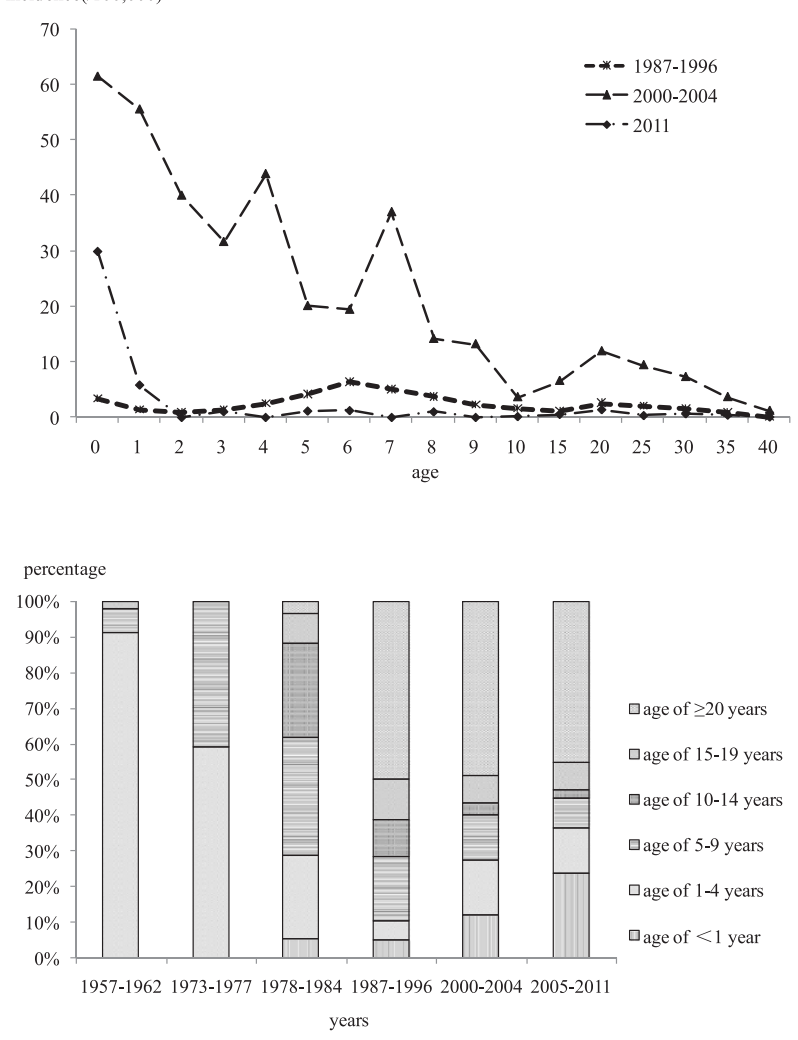
years. Incidence decreased gradually by age. After the introduction of initial planned vaccination in 1978-1984, incidence decreased dramatically from $237.0 / 100,000$ to $465.3 / 100,000$ in children aged $\leq 14$ years. In 1987-1996, incidence declined continually in all age groups, ranging from $0.1 / 100,000$ to $6.4 / 100,000$. Since this period, agespecific incidence showed a change, three peaks of higher incidence were found in infants of $<1$ years, children of 5-7 years and adults of 20-30 years. In 2000-2004, incidence increased in all age groups, ranging from 3.6/ 100,000 to $61.5 / 100,000$ for children aged $\leq 14$ years and $1.1 / 100,000$ to $11.9 / 100,000$ for adults aged $\geq 15$ years, with two crest in infants of $<1$ years and adults of 20-30 years. In 2005-2010, age-specific incidence increased to $5.1 / 100,000-82.9 / 100, .000$ for children aged $\leq 14$ years and $2.3 / 100,000-28.0 / 100,000$ for adults aged $\geq 15$ years, with highest 373.0/100,000 in infants aged $<1$ years. After MV SIA in 2010, incidence declined greatly to $0-1.2 / 100,000$ for $\geq 2$ years age group, while incidence was 29.9/100,000 for $<1$ years age group and 5.8/100,000 for $1-2$ years age group (Figure 3).

Age-proportion distribution of measles cases varied over time. From 1957 to 1962, the most affected age groups were children of 1-4 years old, with proportion of $91.0 \%$. The proportion of 5-9 years was 7.0\%. From 1973 to 1977 , the proportion of measles cases decreased to $59.1 \%$ in children of $1-4$ years, but increased to $40.7 \%$ in children of 5-9 years. From 1978 to 1984, the proportion of 1-4 years and 5-9 years age groups were continually decreased to $23.2 \%$ and $33.0 \%$, respectively. The affected age groups expand to 10-14 years age group with $26.4 \%$ and $\geq 15$ years with $12.0 \%$. In $1987-1996$, the proportion of 1-14 years age group decreased to $5.6 \%$ $18.0 \%$, while the proportion of adults of $\geq 15$ years increased to $61.4 \%$. In 2000-2004, proportion for infants of $<1$ years and children of $1-4$ years increased to $11.8 \%$ and $15.6 \%$, gradually. Adults of $\geq 15$ years accounted for $56.5 \%$. In 2005-2011, proportion of infants aged $<1$ years continually increased to $23.5 \%$. Adults aged $\geq 15$ years accounted for $52.9 \%$ (Figure 3 ).

\section{Spatial distribution of measles incidence}

Measles was observed in all districts in Beijing, while the spatial distribution varied from 1982 to 2011 (Figure 4). In 1982-1985, measles broke out in all districts and the incidence range from 38.3/100,000 to $160.2 / 100,000$. In 1986-1996, measles incidence decreased dramatically in all districts, ranging from $0.2 / 100,000$ to $3.6 / 100,000$. In $1997-2004$, incidence of all districts remained lower level at $0.1 / 100,000$ to $10.5 / 100,000$. In 2005-2010, incidence in all districts increased slightly from $2.1 / 100,000$ to $27.3 / 100,000$. The main high risk districts were Chaoyang, Fengtai and Changping. After MV SIAs in 2010, incidence in all districts declined dramatically to lowest level, which was 0 in three districts.

\section{Seasonality of measles}

Peaks in the reported measles mainly occurred from March to May in 1985-2010, while it was no clear seasonal trend in 2011 when incidence declined to the lowest level (Figure 5).

\section{Vaccination coverage of measles vaccine}

From 1971 to 1977, the MV coverage rate of first dose increased from $71.2 \%$ to $88.6 \%$, while measles incidence remained high level during this period. During period of planned vaccination in 1978-1996, the MV coverage rate of first and second doses increased gradually from $91.2 \%$ to $99.9 \%$, and measles incidence in entire population decreased dramatically from $146.0 / 100,000$ to $0.3 / 100,000$ and incidence in children of $1-14$ years also declined greatly from $616.7 / 100,00$, to $0.1 / 100,000$. During accelerated period of measles control from 1997-2004, the MV coverage rate of second dose appeared a decline around $88.4 \%-94.5 \%$ in $2002-2004$, which was probably related to rebound of measles incidence of whole population and children of 1-14 years in 2005 (Figure 6).

\section{Measles sero-epidemiology}

From 1973 to 1977, antibody positive rate of measles increased from $73.3 \%$ to $95.8 \%$, while incidence decreased from $248.7 / 100,000$ to $42.2 / 100,000$. Antibody positive rate of children 1-4 years was relative low, $63.8 \%-87.5 \%$, when the first dose of MV was administrated at 6 months. Antibody positive rate of children 5-9 years was increased to $84.3 \%-97.4 \%$, when the second and third dose of MV was administrated at 4 and 8 years old. From 1978 to 1996, antibody positive rate increased from the lowest $75.7 \%$ to higher $93.6 \%$, while incidence declined from highest $146.0 / 100,000$ in 1978 to lowest $0.3 / 100,000$ in 1995. Age-specific antibody positive rate of $1-4$ years was increased greatly from $68.0 \%$ to $97.9 \%$ while the first and second dose of MV was administrated at 8 months and 1.5 years old. Antibody positive rate increased from $70.6 \%$ to $98.0 \%$ for $5-14$ years old, while students in Grade 1 and 7 was administrated MV from 1982. From 1997 to 2004 , measles antibody positive rate fall to $93.4 \%$ and $73.4 \%$, while incidence appeared slight rebound. During this period, age-specific antibody positive rate of $1-14$ years was between $85.7 \%$ and $100 \%$, except $68.6 \%-80.0 \%$ in 2003. Range of antibody positive rate in infants $<1$ years was $30.0 \%-61.2 \%$ (Figure 7).

\section{Discussion}

Based on the trend analysis data from previous 60 years in this study, the measures of prevention and control measles at different stages had a tremendous effect on 

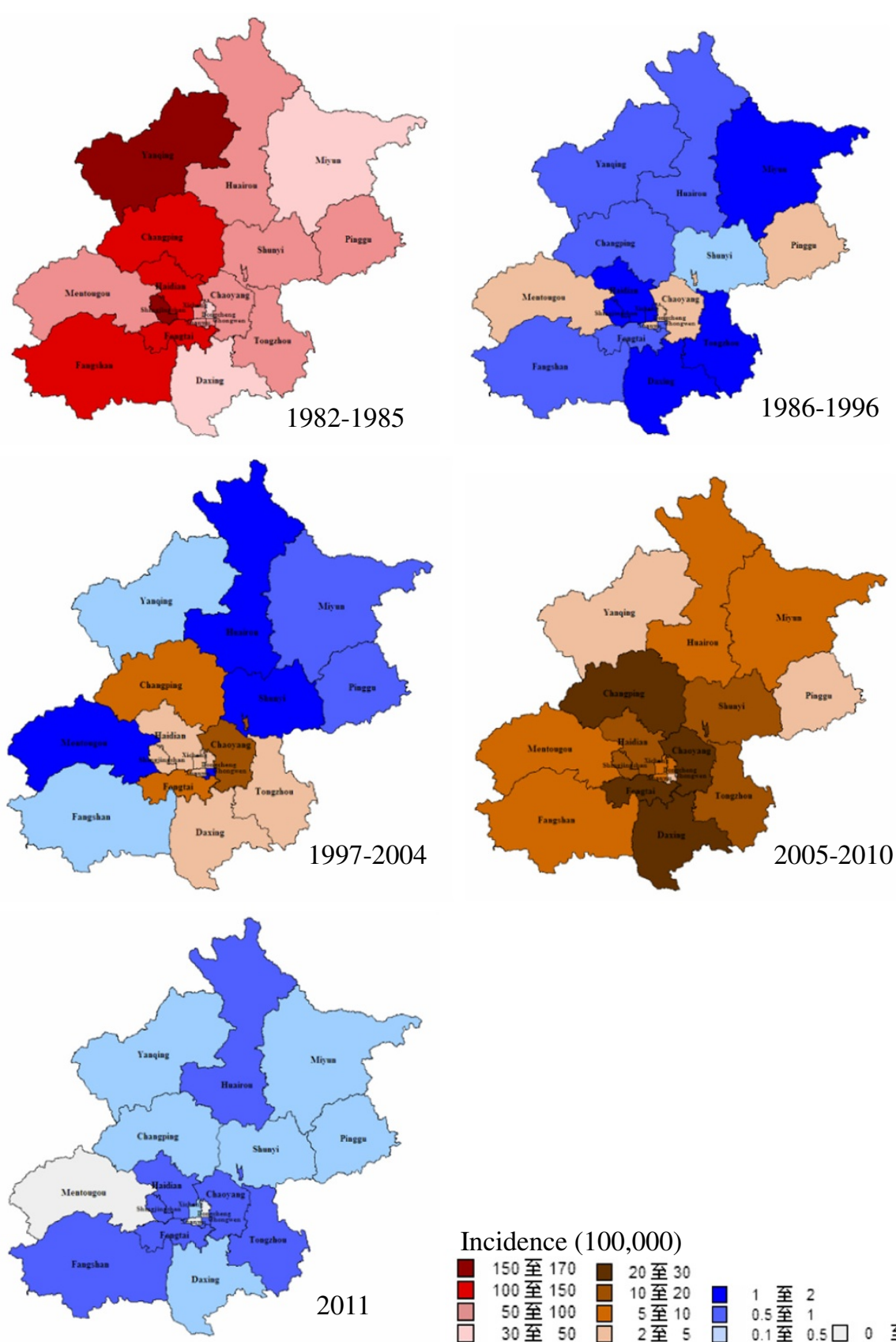

Incidence $(100,000)$

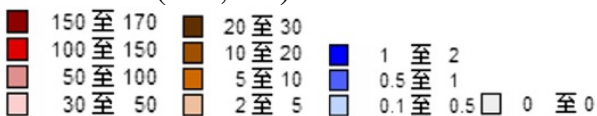

Figure 4 Spatiotemporal distribution of measles incidence in different stages, 1982-2011.

the epidemic dynamics of measles. Consistent with observations from other countries, epidemic cycles of measles occurred every 2-3 years in Beijing before measles vaccination, when measles virus was in a natural infectious state. Virtually everyone experienced measles illness during the childhood [16]. 91\% of individuals were infected by the age of 1-4 years. After implementation of measles vaccination, especially program immunization with two doses at age of 8 months and 1.5 years in 1978, measles incidence drop dramatically $[17,18]$, but resurgences occurred in 1982 and again in 1986. These resurgences may result from low effectiveness of liquid MV from Jing ${ }_{55}$ strains [19] and an accumulation of susceptible to a critical threshold level. This highlighted that situation of lower immunogenicity and administration of two doses was inadequate for the interruption of measles transmission. Jing ${ }_{55} \mathrm{MV}$ strains were replaced by $\mathrm{Hu}_{191}$ strains, which had good immunogenicity and long-term persistence [20] and the third dose was introduced since 1982. Furthermore in 1987, liquid MV was replaced by 


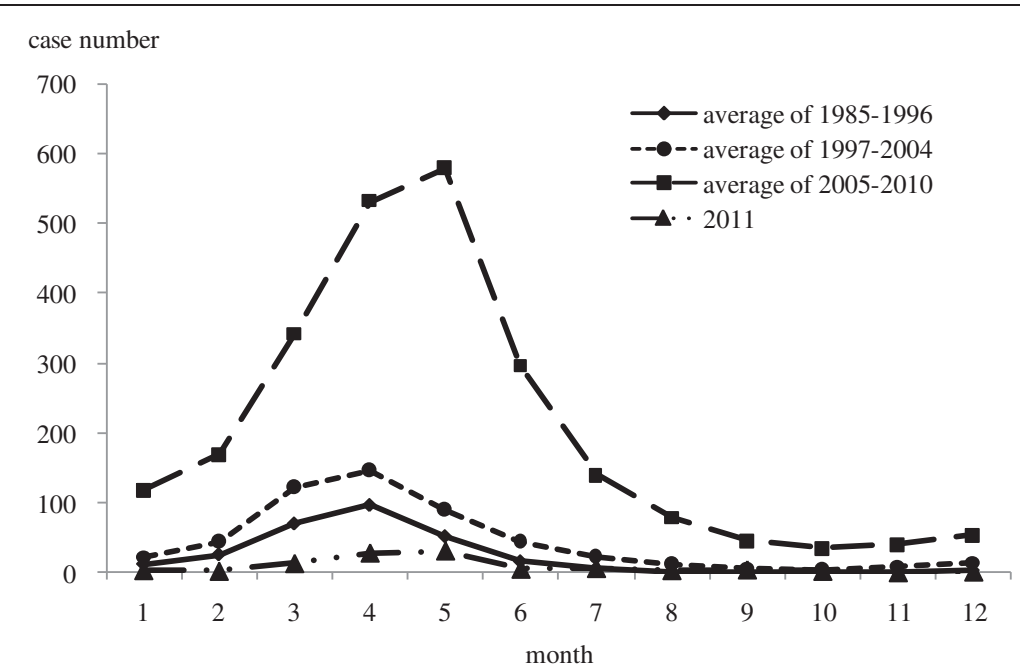

Figure 5 Seasonality of measles in 1985-2011. The reported cases number by month before 1985 was not recorded in the historical documents.

lyophilized vaccine to improve vaccine stability and immunogenicity. Increasing vaccination coverage led to declining incidence of measles to historical lowest level in 1995 and longer inter-epidemic periods of 3-4 years. The incidence of measles appeared rise from 2005 due to enhancement of measles surveillance susceptibility through directly reporting suspected measles cases by network [21]. The 2010 national MV SIAs targeting children aged 8 months to 14 years was successful in largely interrupting measles virus transmission, resulting in almost historical lowest level of measles incidence in 2011.

With the increasing vaccination coverage in Beijing, the age distribution of cases shifted from younger children of 1-4 years to older children of 5-14 years and adults of 15-30 years; The upward shift in the age of measles cases to older age groups with increasing MV coverage was well documented in other settings [22-25]. Toward the end of endemic measles virus transmission in the Americas, outbreaks in some regions included cases among older children and young adults [26,27]. In Beijing, young adults, especially migrant workers from other province living in the poor and crowd environment, clearly played a large role in the initial spread of measles epidemic. China could be divided into 3 groups: eastern, central and western provinces or regions, according to economic and social development. Migrants leaved their hometown in western and central provinces including to Qinghai, Gansu, Yunnan, Sichuan, Hunan, Hubei, Henan,

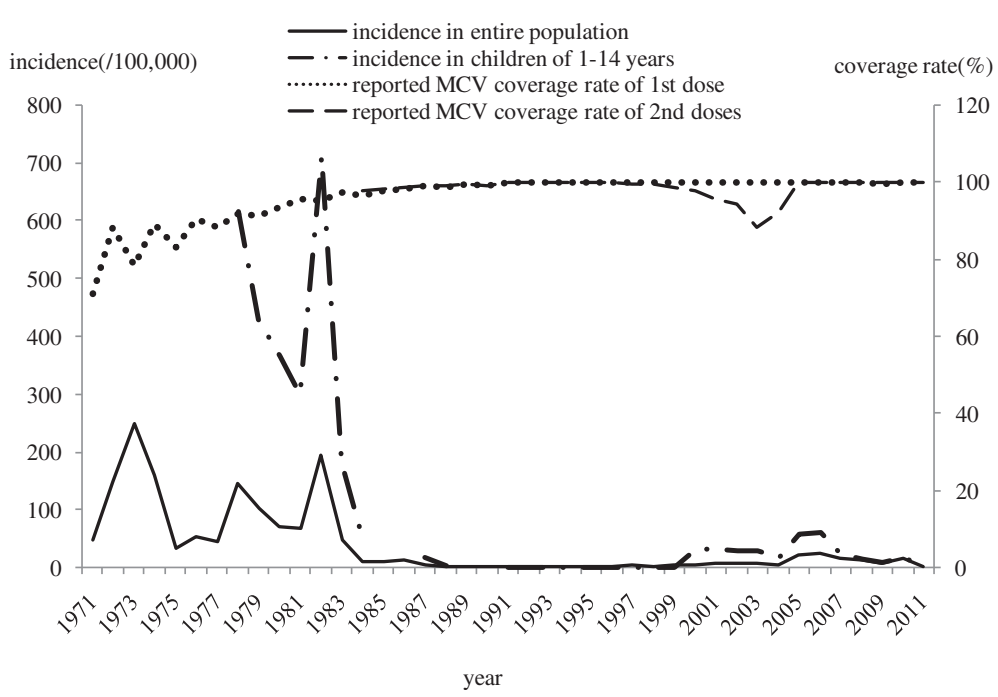

Figure 6 Measles Incidence and immunization coverage rate in Beijing, 1971-2011. 


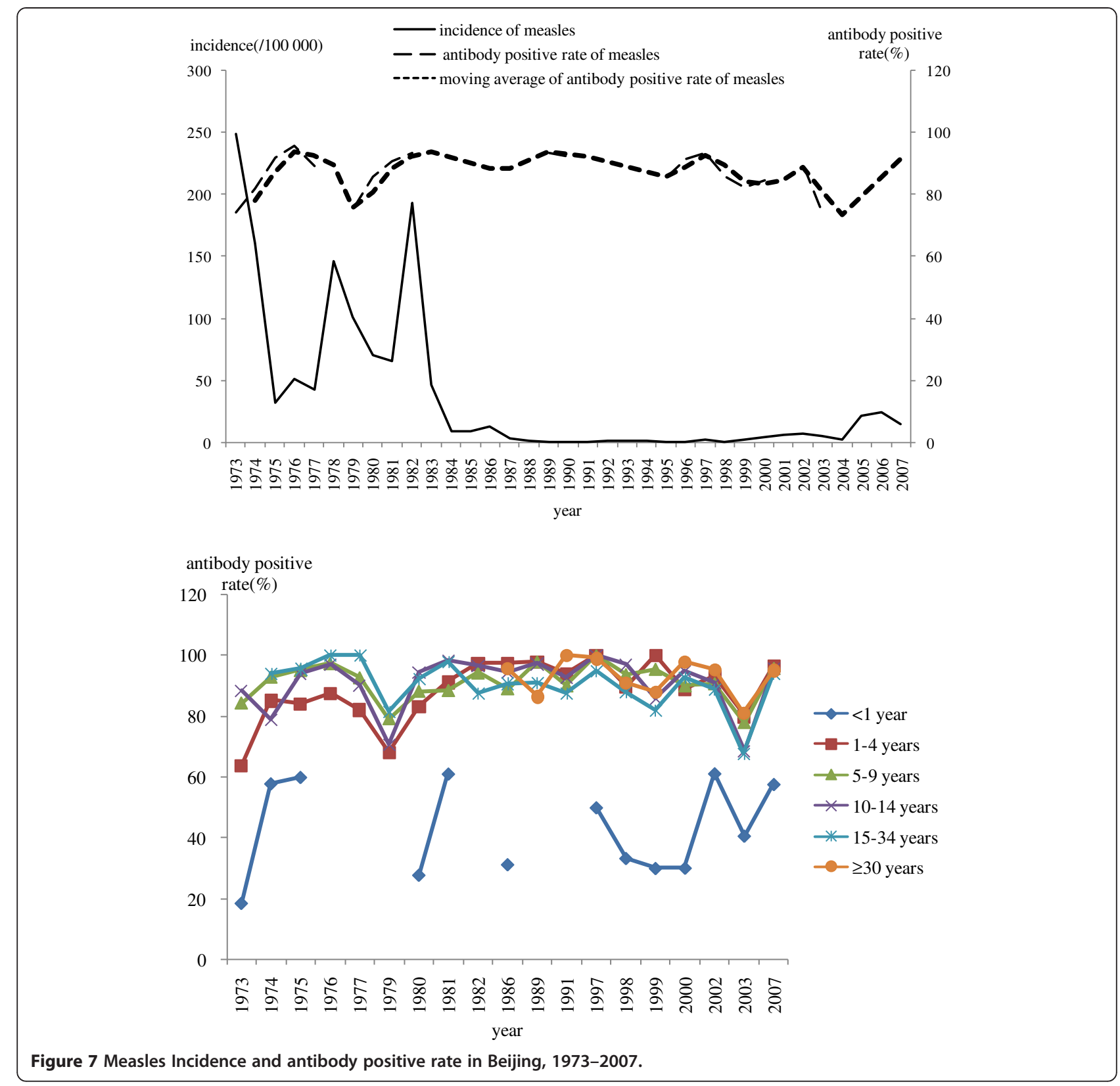

and Anhui, et al. and swarmed to the eastern and more developed provinces including Beijing, Shanghai, Guangdong, Zhejiang, et al. in recent two decades. The national measles surveillance indicated that the average reported measles incidence decreased more in the Western (from $131.2 / 100,000$ to $105.8 / 100,000$ ) than in the Central provinces (from 57.6/100,000 to 55.5/100,000) from 19901999 to 2000-2009. In contrast, incidence in the eastern provinces increased from a lower level of 38.8/100,000 in $1990-1999$ to $65.0 / 100,000$ in 2000-2009. Persons in this age group likely remained susceptible to measles because most were born during the period of MV introduction into the routine immunization program and scaling up of coverage [28]. These young adults born during 1970s1990s were more likely escaped from both natural measles infection and measles vaccination, when measles incidence declined and vaccine coverage was not high during initial period of measles vaccination, especially in other province with lower development of immunization service [29]. Many your adults and migrant population did not have positive measles antibody. It was important to immunize adults susceptible to measles in Beijing. Measles vaccination campaign targeted migrant workers aged 15-40 years was conducted each year since 2005 and had a certain effect on control measles in migrant works in Beijing. Proportion of farmers, workers and farmer workers 
decreased from $17.8 \%$ in 2005 to $9.1 \%$ in 2010 [28]. However, these populations had a strong mobility. Large-scale immunization may not interrupt measles virus transmission. For infants, the age of infection decreased slightly and the proportion of measles case increased gradually with higher vaccination coverage from 1978 in Beijing. Infants born to immune mothers received maternal antibodies transferred during the perinatal period and remained protected, on average, until approximately 4-6 months of age [30]. However, transferred maternal antibodies that were vaccine-induced rather than naturally acquired following measles infection generally existed at lower geometric mean titers in the mother and infant and waned much earlier, leaving the infant unprotected as early as 1 month of age $[31,32]$. In addition, the relatively high incidence among infants and young adults suggested that many young mothers may never have been vaccinated or acquired natural immunity, leaving their newborn children at increased risk from birth because they lacked protective maternal antibody against measles. The outcome of Beijing and other Western Pacific Region countries and areas will help determine whether ensuring high population immunity among adults is necessary to achieve measles elimination in other regions.

Measles incidence has varied widely by district. From 1998 to 2011, the incidence was higher in Chaoyang, Changping and Fengtai districts. Over $50 \%$ of cases were reported from these three districts. The flow of migrant workers from other provinces caused the spread of the disease. These three districts are the suburb in Beijing, in which population increase dramatically with development of economics [33]. The density of the population was higher compared with other districts [34]. Large population size and high population density negatively impact the progress of measles elimination [35]. Increasing numbers of migrant workers could be major reason for the higher incidence of measles.

To interrupt endemic transmission of measles virus, mathematical models indicate that $\geq 93 \%-95 \%$ population immunity is needed [36]. In the region of the Americas, measles was declared eliminated in 2002 after the successful implementation of a strategy that included achieving and sustaining a very high coverage $(\geq 95 \%)$ of children [37]. Application of similar strategies in 7 southern African countries led to similar results [38]. In Beijing, the coverage of first dose MV increased steadily and reached to almost $100 \%$ from 1990. However, a gap remained between the second dose coverage and the 95\% goal in 2002-2004 while SARS happened in China. The fact that the resurgence of measles incidence was found in 2005, especially for age of 1-6 years also indicated that routine coverage was insufficient to interrupt measles transmission in this age group. In addition, MV coverage rates in migrant children were only $76.6 \%-81.6 \%$ through investigation in 1998-2003, which significantly lower than that in local children [39-41]. SIAs among migrant children under 7 years old was implemented since 2000 in Beijing. The immunization management for migrant children after SIAs was improved greatly. However, the migrant children who lived in populous townships or had shorter residence time were still the emphasis [42-44]. Strong commitment and effort were urgently needed to maintain $95 \%$ of 2 doses routine coverage and measure coverage accurately.

This study had some limitations. Firstly, the reported MV coverage used in our analysis was likely higher than the actual MV coverage in some areas [45-47]. Secondly, it is acknowledged that due to the lack of the annual population structure in the earlier period (1957-1996), average incidence was calculated assuming the same population denominators by age group in the same stage (1957-1962, 1978, 1984, 1987-1996). Thirdly, convenience sampling was used in sero-epidemiology surveys, and the population selected may bias distribution of measles antibody level.

\section{Conclusions}

This study showed the various effects of different strategies on the incidence of measles in Beijing, China, which would provide valuable information to commit local public health policy making and could be applied at national level. There remains a need to fully implement all components of the measles control strategy, to consider finetuning the strategies to sustain recent gains in measles incidence reduction and to make further progress. The emphasis is on implementing: (1) strategy to increase measles vaccine coverage in migrant adults and migrant children if transmission persists in these groups (2) routinely monitoring and validating reported vaccination coverage; (3) further research needed to better understand the changing measles epidemiology, including the role that young infants and older age groups played in sustaining measles virus transmission.

\section{Consent}

Written informed consent was obtained from the patient's guardian/parent/next of kin for the publication of this report and any accompanying images.

\section{Competing interests}

The authors declare that they have no competing interests.

\section{Authors' contributions}

$J L:$ (1) conception and design of the study, (2) acquisition, analysis and interpretation of data, (3) draft of the article and selection of manuscripts to discuss the results, (4) final approval of the version to be submitted; LL, XHP and MPS: (1) revising it critically for important intellectual content, (2) discussing the results, (4) final approval of the version to be submitted; RM and DLL: (1) acquisition of data, (2) analysis and interpretation of data, (3) final approval of the version to be submitted; JW: (1) revising it critically for important intellectual content, (2) editing for corrections in the English 
quality, (3) final approval of the version to be submitted. All authors read and approved the final manuscript.

\section{Acknowledgments}

We acknowledge the great efforts of the immunization officers, surveillance officers and measles laboratory staff throughout Beijing. We also thank all levels of governments for providing financial and operational support, leading to measles incidence and mortality reduction, and progress toward the measles elimination goal.

Received: 17 February 2013 Accepted: 11 October 2013

Published: 21 October 2013

\section{References}

1. CDC: Global measles control and regional elimination, 1998-1999. MMWR 1999, 48(49):1124-1130.

2. Guo JY: Beijing historical materials of health and epidemic prevention- in commemoration of the 50 anniversary of the founding of the People's Republic of China. 1st edition. Beijing: Beijing Press; 1999.

3. Ma JQ: Effect of development of Interneton infectious disease reporting system. Chin.J of PHM 2003, 19(3):245-247.

4. Pan EC: Analysis on present reporting system from the prevalence of SARS. Chin J Dis Control Prev 2004, 5(8):435-437.

5. Ze WY, Diao LD, Xu AQ: Expanded program on immunization. 2nd edition. Shanghai: Shanghai Publishing House of Scientific and Technological Literature; 2001.

6. WHO: Progress towards the 2012 measles elimination goal in WHO's Western Pacific Region, 1990-2008. Wkly Epidemiol Rec 2009, 84(27):271-279.

7. Guris D: Module on best practices for measles surveillance: Department of Vaccines and Biologicals. World Health Organization; 2001.

8. Li X, Kang D, Zhang Y, Wei G, Liu W, Fang L, Yang H, Cao W: Epidemic trend of measles in Shandong Province, China, 1963-2005. Public Health 2012, 126(12):1017-1023.

9. Ma C, An Z, Hao L, Cairns KL, Zhang Y, Ma J, Cao L, Wen N, Xu W, Liang X, et al: Progress toward measles elimination in the People's Republic of China, 2000-2009. J Infect Dis 2011, 204(Suppl 1):S447-S454.

10. Rota JS, Heath JL, Rota PA, King GE, Celma ML, Carabana J, FernandezMunoz R, Brown D, Jin L, Bellini WJ: Molecular epidemiology of measles virus: identification of pathways of transmission and implications for measles elimination. J Infect Dis 1996, 173(1):32-37.

11. CDC: Rubella Laboratory Network, January 2004-June 2005. MMWR 2005, 54:1100-1104.

12. WHO: Global measles and rubella strategic plan, 2012-2020. Geneva (Switzerland) 2012:1-44.

13. Papania MJ, Seward JF, Redd SB, Lievano F, Harpaz R, Wharton ME: Epidemiology of measles in the United States, 1997-2001. J Infect Dis 2004, 189(Suppl 1):S61-S68.

14. WHO: WHO-recommended standards for surveillance of selected vaccinepreventable diseases. Department of Vaccines and Biologicals, Health Technology and Pharmaceuticals, World Health Organization; 2003.

15. Sniadack DH, Mendoza-Aldana J, Jee $Y$, Bayutas B, Lorenzo-Mariano KM: Progress and challenges for measles elimination by 2012 in the Western Pacific Region. J Infect Dis 2011, 204(suppl 1):S439-S446.

16. Edmunds WJ, Gay NJ, Kretzschmar M, Pebody RG, Wachmann H: The pre-vaccination epidemiology of measles, mumps and rubella in Europe: implications for modelling studies. Epidemiol Infect 2000, 125(3):635-650.

17. Lepow ML: Measles vaccine and measles control. Pediatr Ann 1990, 19(9):543-545. 548-550.

18. Dabis F, Waldman RJ, Sow A, Jones TS, Madzou G: Measles control in Africa. Lancet 1986, 2(8499):162-163.

19. Diao LD, Xu AQ, Feng ZJ, Wang H: Measles. 1st edition. Shanghai: Shanghai Press of Science and Technology Document; 2001.

20. Chen ZH, Ren XG, Yang HY, Wu T, Xun WQ, Han MM, Hu FJ, Xu BX, Chen HJ, Chu BL: Study on the Sero-Epidemiologic Efficacy of Shanghai 191 Measles Live Vaccine after 16 Years since Primary Immunization. Chinese Journal of Biologicals 1989, 2(7):5-9.

21. Yang P, Duan W, Lv M, Shi W, Peng X, Wang X, Lu Y, Liang H, Seale H, Pang $X$, et al: Review of an influenza surveillance system, Beijing, People's Republic of China. Emerg Infect Dis 2009, 15(10):1603-1608.
22. Cherry JD: The 'new' epidemiology of measles and rubella. Hosp Pract 1980, 15(7):49-57.

23. Duclos P, Redd SC, Varughese P, Hersh BS: Measles in adults in Canada and the United States: implications for measles elimination and eradication. Int J Epidemiol 1999, 28(1):141-146.

24. Goodson JL, Masresha BG, Wannemuehler K, Uzicanin A, Cochi S: Changing epidemiology of measles in Africa. J Infect Dis 2011, 204(Suppl 1):S205-S214.

25. Chen RT, Weierbach R, Bisoffi Z, Cutts F, Rhodes P, Ramaroson S, Ntembagara C, Bizimana F: A 'post-honeymoon period' measles outbreak in Muyinga sector, Burundi. Int J Epidemio/ 1994, 23(1):185-193.

26. Hersh BS, Tambini G, Nogueira AC, Carrasco P, de Quadros CA: Review of regional measles surveillance data in the Americas, 1996-99. Lancet 2000, 355(9219):1943-1948.

27. de Quadros CA, Izurieta H, Venczel L, Carrasco P: Measles eradication in the Americas: progress to date. J Infect Dis 2004, 189(Suppl 1):S227-S235.

28. Li J, Lu L, Liu DL, Ma R, Wu J, Pang XH: Measles epidemiology and elimination measure evaluation in Beijing, 2005-2010. Disease Surveilance 2012, 5(27):353-357.

29. Sniadack DH, Mendoza-Aldana J, Huyen DT, Van TT, Cuong NV, Olive JM, Toda K, Hien NT: Epidemiology of a measles epidemic in Vietnam 20082010. J Infect Dis 2011, 204(Suppl 1):S476-S482.

30. Ferrari MJ, Grais RF, Bharti N, Conlan AJ, Bjornstad ON, Wolfson LJ, Guerin PJ, Djibo A, Grenfell BT: The dynamics of measles in sub-Saharan Africa. Nature 2008, 451(7179):679-684.

31. Cutts FT, Markowitz LE: Successes and failures in measles control. J Infect Dis 1994, 170(Suppl 1):S32-S41.

32. Leuridan $E$, Hens $N$, Hutse V, leven M, Aerts M, Van Damme P: Early waning of maternal measles antibodies in era of measles elimination: longitudinal study. BMJ 2010, 340:1626-1633.

33. Champion AG: A Changing Demographic Regime and Evolving Poly centric Urban Regions: Consequences for the Size, Composition and Distribution of City Populations. Urban Studies 2001, 38(4):657-677.

34. Feng J, Zhou Y: The growth and distribution of population in Beijing metropolitan area (1982-2000). ACTA GEOGRAPHICA SINICA-CHINESE EDITION- 2003, 58(6):903-916.

35. Yoshikura H: Negative impacts of large population size and high population density on the progress of measles elimination. Jpn J Infect Dis 2012, 65(5):450-454.

36. Anderson RM, May RM: Vaccination and herd immunity to infectious diseases. Nature 1985, 318(6044):323-329.

37. de Quadros CA, Olive JM, Hersh BS, Strassburg MA, Henderson DA, Brandling-Bennett D, Alleyne GA: Measles elimination in the Americas. Evolving strategies. JAMA 1996, 275(3):224-229.

38. Biellik R, Madema S, Taole A, Kutsulukuta A, Allies E, Eggers R, Ngcobo N, Nxumalo M, Shearley A, Mabuzane E, et al: First 5 years of measles elimination in southern Africa: 1996-2000. Lancet 2002, 359(9317):1564-1568.

39. Liu AH, Gu YH, Xu SX: Investigation on Immunization Coverage Rate of Floating Children in Beijing. Chinese Journal of Vaccines and Immunization 2000, 5(6):263-266.

40. Sun MP, Liu DW, Liu AH, Liu DL, Fan CY, Miao L, Lu L, Zhang XC, Da-we i LIU, Ai-hua LIU, et al: (Beijing Munici ple Center for Disease Prevention and Control, Beijing 100013, China); Investigation of Immunization Coverage Rate of Children Living in Floating Population Area and the Affecting Factors [J]. Chinese Journal of Vaccines and Immunization 2002, 2(8):91-94.

41. LIU DW, SUN MP, LIU WX, Fan CH, Lu L, Liu DL: Comparative Study on Immunization Coverage Rates of Nine Vaccines Between Local and Floating Children [J]. Chinese Journal of Vaccines and Immunization 2007, 2(13):165-169

42. Yu X, LU X, Wu B: Management on EPI in Floating Children. Chinese Journal of Vaccines and Immunization 2005, 1:38-42.

43. SUN MP, MA R, ZENG Y, Lu L, Liu DL, Wang LY, Liu WX: Effect Evaluation of Supplementary Immunization Activities Among Migrant Children Under 7 Years Old in Beijing. Chinese Journal of Vaccines and Immunization 2008, 6(14):543-545

44. MA R, SUN MP, ZENG Y, Lu L, Liu DL, Wang LY, Liu WX, Zhang Q, Xu RH, Huang ST, et al: Implementing and Effect Evaluation of Supplementary Immunization Activities Among Migrant Children Under 7 Years Old in Beijing in 2007 [J]. Chinese Journal of Vaccines and Immunization 2007, 6(13):581-585. 
45. Zuo SY, Wang XJ, Cao L, Zhang LX, Zhang RZ: Quality of reports of routine immunization coverage and its affecting factors. Chinese Journal of Vaccines and Immunization 2002, 6(8):337-340.

46. Mavimbe JC, Braa J, Bjune G: Assessing immunization data quality from routine reports in Mozambique. Bmc Public Health 2005, 5(1):108-115.

47. Onta SR, Sabroe $S$, Hansen EH: The quality of immunization data from routine primary health care reports: a case from Nepal. Health Policy Plann 1998, 13(2):131-139.

doi:10.1186/1471-2458-13-986

Cite this article as: Li et al:: A 60-year review on the changing

epidemiology of measles in capital Beijing, China, 1951-2011. BMC Public Health 2013 13:986.

\section{Submit your next manuscript to BioMed Central and take full advantage of:}

- Convenient online submission

- Thorough peer review

- No space constraints or color figure charges

- Immediate publication on acceptance

- Inclusion in PubMed, CAS, Scopus and Google Scholar

- Research which is freely available for redistribution 